\title{
MODELOS DINÁMICOS Y LA INGENIERÍA DEL SOFTWARE
}

Ing. Rosmeri Mayta Huatuco

Ing. Víctor Pérez Quispe

RESUMEN

En el presente estudio, se analiza el proceso de desarrollo del software (PDS), como un conjunto de herramientas, métodos y prácticas que permiten producir software. Se presenta modelos dinámicos para simular entornos de desarrollo en una organización.

\section{ABSTRACT}

Presently study, the process of development of the software is analyzed (PDS), as a group of tools, methods and practical that allows to produce software. It is presented dynamic models to simulate development environments in an organization.

\section{INTRODUCCIÓN}

El proceso de desarrollo de software puede definirse como un conjunto de herramientas, métodos y prácticas que se emplean para producir software. Como cualquier otra organización, las dedicadas al desarrollo de software mantienen entre sus principales fines, la producción de software de acuerdo con la planificación inicial realizada, además de una constante mejora con el fin de lograr los tres objetivos últimos de cualquier proceso de producción: alta calidad y bajo coste, en el mínimo tiempo. La gestión de un PDS engloba, por tanto, todas las funciones que mantengan a un proyecto dentro de unos objetivos de coste, calidad y duración previamente estimados. La mayoría de estas funciones y técnicas de gestión y control empleadas, se han importado de otras industrias de producción que desarrollaron estos métodos a principios de siglo.

Sin embargo, los problemas de gestión que surgen en las organizaciones de desarrollo provienen principalmente de la gestión estratégica, como puede ser el entorno socio/político de la organización, su nivel de madurez y los factores humanos o del personal técnico. De hecho, la incapacidad de las técnicas de gestión actuales para tratar el complicado factor humano ha sido reconocida como uno de los principales problemas.

A principios de los 90 se produce un salto significativo en la gestión de Proyectos de Desarrollo de Software (PDS) con la aparición del primer Modelo Dinámico [Abdel-Hamid y Madnick, 1991]. Este modelo incorpora las actividades básicas relacionadas con la de gestión de recursos humanos, producción de software, planificación y control.

Las características de esta disciplina contribuyen a que se desarrollen modelos del proceso de desarrollo de software que permiten manejar complejidades dinámicas y conocer los efectos secundarios de las decisiones tomadas sobre la globalidad del mismo, aspectos que son esenciales para desarrollar una buena estrategia. Para nosotros un PDS es un sistema dinámico socio-tecnológico complejo, cuya evolución temporal viene dada por su estructura interna, por las relaciones existentes entre el personal técnico que trabaja en el mismo y por el nivel de madurez de la propia organización de desarrollo.

\footnotetext{
“La gestión de

un PDS

engloba, por

tanto, todas las

funciones que

mantengan a

un proyecto

dentro de unos

objetivos de

coste, calidad y duración

previamente estimados."
} 


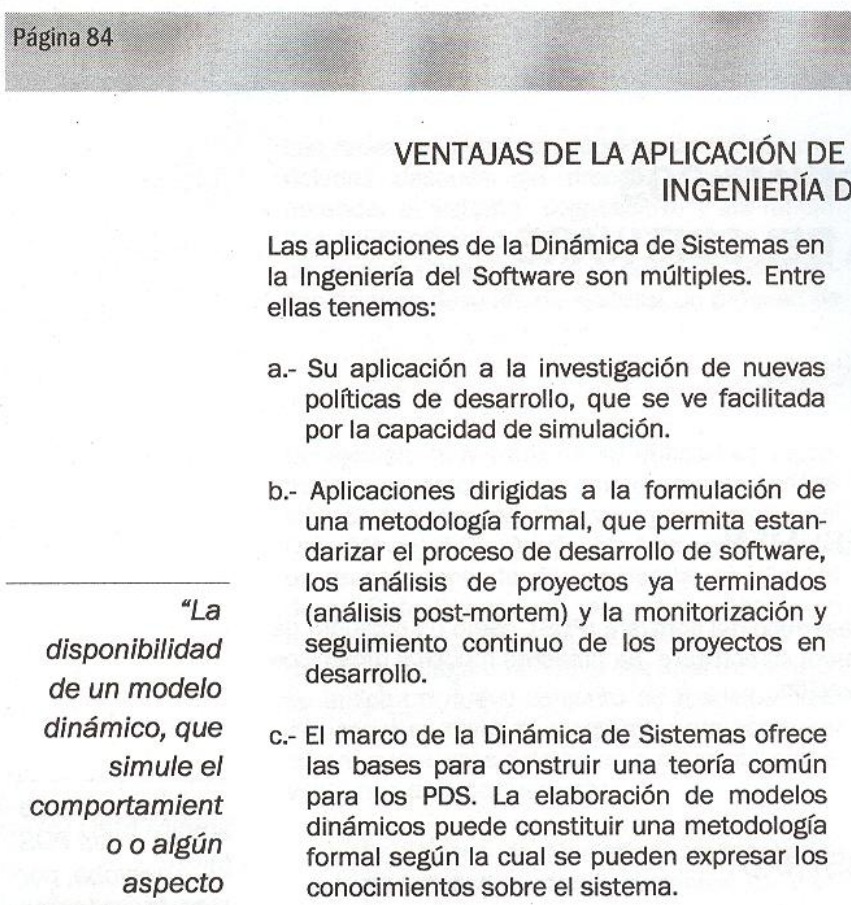

concreto de una

organización,

constituye una

herramienta

fundamental en

la toma de

decisiones de

dicha

organización."

A continuación, se presentan algunos modelos dinámicos cuyas aportaciones fundamentales se centran en añadir nuevas capacidades y aplicaciones al Modelo de Abdel-Hamid y Madnick. Estos modelos se pueden dividir en dos grandes grupos:

1. Los de carácter general creados para simular entornos específicos de desarrollo dentro de una determinada organización. Entre estos modelos destacan:

- El Modelo SEPS (Software Engineering Process Simulation). Elaborado en el laboratorio JPL (Jet Propulsion Laboratory). Diseñado para simular el comportamiento de proyectos grandes considerando la existencia de un doble ciclo de vida: el proceso de desarrollo propiamente dicho y el proceso de toma de decisiones. Además, introduce sistemas expertos con lógica fuzzy en la interfaz del modelo.

- El Modelo de Draper Laboratory. El Modelo de Draper constituye una ampliación del Modelo de Abdel-Hamid y Madnick. Presenta como novedad la incorporación de la etapa de Análisis de Requisitos (no tratada en el Modelo de Abdel-Hamid y Madnick) contemplando la posibilidad de que estos requisitos puedan cambiar a lo
-El proceso de construcción del modelo, por sí mismo, obliga a los investigadores a tener un alto conocimiento de cuáles son los parámetros claves que influyen en el comportamiento del sistema y cómo se relacionan entre sí constituyendo lazos de realimentación.

e.- Por otro lado, el potencial de los modelos de simulación para la formación y el entrenamiento de los directores de proyectos es manifiesto: los entornos de simulación sitúan a los directores frente a situaciones reales que pueden encontrar en la práctica y les permite adquirir experiencia sin correr riesgos.

f.- La disponibilidad de un modelo dinámico, que simule el comportamiento o algún aspecto concreto de una organización, y un entorno de simulación potente como los que existen en la actualidad, constituye una herramienta fundamental en la toma de decisiones de dicha organización. blemas concretos presentados en la gestión de PDS. Entre estos modelos destacan:

a.- El Modelo de Chichacly. Este modelo realizado para estudiar el impacto de un cambio de tecnología, concretamente el cambio de $\mathrm{C}$ a $\mathrm{C}++$, dentro de una empresa de desarrollo de aplicaciones para Macintosh poniendo de manifiesto la reacción de oposición o resistencia por parte de los técnicos ante la implantación de cualquier tipo de cambio en las técnicas o métodos de trabajo empleados normalmente.

b.-El Modelo Aranda/Friddaman//Oliva. Este modelo añade aspectos nuevos al modelo de Abdel-Hamid y Madnick como son los efectos del empleo de las técnicas TQM (Total Quality Management) para el control de calidad, la ampliación del horizonte temporal del proyecto con el fin de recoger las diferentes versiones del software producido y el estudio del impacto comercial del producto final. 
c.- El modelo Multiproyecto. Este modelo estudia las transferencias netas de personal que se producen cuando interactúan dos proyectos por los mismos recursos.

3. Diseño de una base histórica de PDS reales.

Trabajos que deberían afrontarse en un futuro serían los siguientes:

- Desarrollo de un Modelo Dinámico Multiproyecto basándonos en los trabajos realizados por Abdel-Hamid sobre este tema.

- Aplicación de técnicas de aprendizaje automático para obtener reglas de gestión antes y durante el proceso de desarrollo de software para optimizar la calidad del producto final y el coste y tiempo de entrega del proyecto.

- Diseño de un Modelo Dinámico Avanzado que pueda aplicarse por separado en cada una de las etapas básicas del proceso de desarrollo de software recogiendo las singularidades de cada una de ellas y que, al mismo tiempo, puedan acoplarse los resultados obtenidos en cada etapa para analizar la evolución global del proyecto.

- Poder obtener a partir de un Modelo Dinámico Avanzado un sistema de generación de métricas para cada etapa básica.

\section{REFERENCIAS BÁSICAS}

- [Abdel-Hamid y Madnick, 1991] AbdelHamid T. K., Madnick S. E., Software Project Dynamics: An Integrated Approach, Englewood Cliffs, NJ: Prentice-Hall 1991.
- Aracil J., Gordillo, F. Dinámica de Sistemas. Alianza Editorial, Madrid 1997.

\section{REFERENCIAS SOBRE MODELOS DINÁMICOS}

- Abdel-Hamid T. K., Thinking in Circles.

- Aranda R., Friddaman T., Oliva R., Quality MicroWorlds: Modeling the impact of quality initiatives over the software product life cycle.

- Chichakly K., The Bifocal Vantage Point. Managing software projects from a systems thinking perspective.

- Dhiedl E. W., Strategy-support software to enhance executive dialog and debate.

- Lin C. Y., Walking on Battlefields: Tools for Strategic software mangement.

- Smith B. J., Nguyen N., Vidale R., Death of a software manager: How to avoid career suicide through dynamic software process modeling.

- Hamid T. K., A Multiproject Perspectiv of Single-Project Dynamics, J. Systems Software, 1993.
- Hansen G. A., Simulating Software Deve lopment Processes, IEEE Computer 1996.

- Ramos I., Riquelme J. C., Obtención de reglas de gestión para proyectos de desarrollo de software, III Jornadas de Ingeniería del Software, Murcia, pp.: $387-$ 398, 1998.

- Rodrigues A. G., Williams T. M., System dynamics in software project management: towards the development a formal a integrated framework, European Journel of Information systems, $n^{\circ} 6$, pp.: 51 66, 1997.

- Tvedt J. D., A System Dynamics Model of the Software Inspection Process, Technical Report TR-95-007, Computer Science and Engineering Department Arizona State University, Tempe. Arizona,1995.

- of Software Projects, Software - Concepts and Tools, 1994. 